\title{
An inductive approach to Coxeter arrangements and Solomon's descent algebra
}

\author{
J. Matthew Douglass • Götz Pfeiffer • \\ Gerhard Röhrle
}

Received: 4 April 2011 / Accepted: 13 June 2011 / Published online: 20 July 2011

(C) Springer Science+Business Media, LLC 2011

\begin{abstract}
In our recent paper (Douglass et al. arXiv:1101.2075 (2011)), we claimed that both the group algebra of a finite Coxeter group $W$ as well as the Orlik-Solomon algebra of $W$ can be decomposed into a sum of induced one-dimensional representations of centralizers, one for each conjugacy class of elements of $W$, and gave a uniform proof of this claim for symmetric groups. In this note, we outline an inductive approach to our conjecture. As an application of this method, we prove the inductive version of the conjecture for finite Coxeter groups of rank up to 2 .
\end{abstract}

Keywords Coxeter groups $\cdot$ Reflection arrangements $\cdot$ Descent algebra $\cdot$ Dihedral groups

\section{Introduction}

Let $W$ be a finite Coxeter group, generated by a set $S$ of simple reflections. If $|S|=r$, then $W$ acts as a reflection group on Euclidean $r$-space $V$. The reflection arrangement of $W$ is the hyperplane arrangement consisting of the reflecting hyperplanes in $V$ of all the reflections in $W$. The Orlik-Solomon algebra $A(W)$ of $W$ is the cohomology

\footnotetext{
J.M. Douglass

Department of Mathematics, University of North Texas, Denton, TX 76203, USA

e-mail: douglass@unt.edu

G. Pfeiffer

School of Mathematics, Statistics and Applied Mathematics, National University of Ireland, Galway, University Road, Galway, Ireland

e-mail: goetz.pfeiffer@nuigalway.ie

G. Röhrle ( $\varangle)$

Fakultät für Mathematik, Ruhr-Universität Bochum, 44780 Bochum, Germany

e-mail: gerhard.roehrle@rub.de
} 
ring of the complement of the complexified reflection arrangement. It follows from a result of Brieskorn [3] that the algebra $A(W)$ is a $W$-module of dimension $|W|$. For some history of the computation of $A(W)$ as a $W$-module, see the introduction of our recent paper [4].

In [4], we claimed that both the group algebra $\mathbb{C} W$ of $W$ (affording the regular character $\rho_{W}$ ) as well as the Orlik-Solomon algebra $A(W)$ (affording the OrlikSolomon character $\omega_{W}$ ) can be decomposed into a sum of induced one-dimensional representations of centralizers, one for each conjugacy class of elements of $W$, in the following interlaced way.

Conjecture A Let $\mathcal{R}$ be a set of representatives of the conjugacy classes of $W$. Then, for each $w \in \mathcal{R}$, there are linear characters $\widetilde{\varphi}_{w}$ and $\widetilde{\psi}_{w}$ of $C_{W}(w)$ such that

$$
\rho_{W}=\sum_{w \in \mathcal{R}} \operatorname{Ind}_{C_{W}(w)}^{W} \tilde{\varphi}_{w}, \quad \omega_{W}=\sum_{w \in \mathcal{R}} \operatorname{Ind}_{C_{W}(w)}^{W} \widetilde{\psi}_{w}
$$

are sums of induced linear characters. Moreover, for each $w \in \mathcal{R}$, the characters $\widetilde{\varphi}_{w}$ and $\widetilde{\psi}_{w}$ can be chosen so that

$$
\widetilde{\psi}_{w}=\widetilde{\varphi}_{w} \in \alpha_{w},
$$

where $\epsilon$ is the sign character of $W$, and $\alpha_{w}$ is the determinant on the 1-eigenspace of $w$.

When $W$ is a symmetric group, the formula for $\rho_{W}$ has been proved independently by Bergeron, Bergeron, and Garsia [1], Hanlon [6], and Schocker [14]. The formula for $\omega_{W}$ follows from work of Lehrer and Solomon [9], who also checked the identity for $\omega_{W}$ in the case of a dihedral group $W$. Conjecture 2.1 in [4] is a graded refinement of Conjecture A and the main result in [4] is a uniform proof of this refined conjecture for symmetric groups.

The details of the proof of Conjecture 2.1 in [4] for symmetric groups rely on properties of these groups not shared by other finite Coxeter groups. However, the underlying strategy of the proof using induced characters both generalizes and admits a "relative" version, for pairs $\left(W, W_{L}\right)$, where $W_{L}$ is a parabolic subgroup of $W$. In Sect. 4, we formalize this notion in Conjecture $C$, show how it leads to a proof of Conjecture A, and describe a two-step procedure that can be used to prove this relative conjecture. Prior to that, in Sects. 2 and 3 we review some notation and basic facts about the descent algebra $\Sigma(W)$ and the Orlik-Solomon algebra $A(W)$. In the final section, we apply the methods from Sect. 4 and prove Conjecture $\mathrm{C}$ for all pairs $\left(W, W_{L}\right)$ where $W$ is arbitrary and $W_{L}$ has rank at most 2 . As a consequence, we deduce that Conjecture A holds for Coxeter groups of rank 2 or less.

\section{Minimal length transversals of parabolic subgroups}

The descent algebra of a finite Coxeter group $W$ encodes many aspects of the combinatorics of the minimal length coset representatives of the standard parabolic subgroups of $W$. In this section, we provide notation and summarize useful properties of these distinguished coset representatives following Pfeiffer [12]. 
For $J \subseteq S$, let

$$
X_{J}=\{w \in W: \ell(s w)>\ell(w) \text { for all } s \in J\} .
$$

Then $X_{J}$ is a right transversal of the parabolic subgroup $W_{J}=\langle J\rangle$ of $W$, consisting of the unique elements of minimal length in their cosets. If we set

$$
x_{J}=\sum_{x \in X_{J}} x^{-1} \in \mathbb{C} W
$$

then, by Solomon's Theorem [15], the subspace

$$
\Sigma(W)=\left\langle x_{J}: J \subseteq S\right\rangle_{\mathbb{C}}
$$

is a $2^{r}$-dimensional subalgebra of the group algebra $\mathbb{C} W$, called the descent algebra of $W$.

For $J \subseteq S$, denote

$$
X_{J}^{\sharp}=\left\{x \in X_{J}: J^{x} \subseteq S\right\} .
$$

The action of $W$ on itself by conjugation partitions the power set of $S$ into equivalence classes of $W$-conjugate subsets. We call the class

$$
[J]=\left\{J^{x}: x \in X_{J}^{\sharp}\right\}
$$

of a subset $J \subseteq S$ the shape of $J$, and denote by

$$
\Lambda=\{[J]: J \subseteq S\}
$$

the set of shapes of $W$. The shapes parametrize the conjugacy classes of parabolic subgroups of $W$, since two subsets $J, K \subseteq S$ are conjugate if and only if the corresponding parabolic subgroups $W_{J}$ and $W_{K}$ are conjugate. We say that a parabolic subgroup of $W$ has shape $[J]$ if it is conjugate to $W_{J}$ in $W$.

Furthermore, for $J \subseteq S$, we define

$$
N_{J}=\left\{x \in X_{J}: J^{x}=J\right\} .
$$

Then $N_{J}$ is a subgroup of $W$ and by results of Howlett [7], the normalizer of $W_{J}$ in $W$ is a semi-direct product $N_{W}\left(W_{J}\right)=W_{J} \rtimes N_{J}$.

An element $w \in W$ is called cuspidal in case $w$ has no fixed points in the reflection representation of $W$. Thus, for $J \subseteq S$, an element $w \in W_{J}$ is cuspidal in the parabolic subgroup $W_{J}$ if $w$ has no fixed points in the orthogonal complement of $\operatorname{Fix}\left(W_{J}\right)$ in $V$, where $\operatorname{Fix}\left(W_{J}\right)$ is the fixed point subspace of $W_{J}$ in $V$. If $w$ is a cuspidal element in $W_{J}$, then the quotient $C_{W}(w) / C_{W_{J}}(w)$ is isomorphic to $N_{J}$ (see [8]).

We consider the character $\alpha_{J}$ of $N_{W}\left(W_{J}\right)$, defined, for $w \in N_{W}\left(W_{J}\right)$, as

$$
\alpha_{J}(w)=\operatorname{det}\left(\left.w\right|_{\operatorname{Fix}\left(W_{J}\right)}\right)
$$

Note that $W_{J}$ is contained in the kernel of $\alpha_{J}$ and so $\alpha_{J}(u n)=\alpha_{J}(n)$ for $u \in W_{J}$, $n \in N_{J}$. 
Lemma 2.1 Let $J \subseteq S$. For $n \in N_{J}$ denote by $\sigma_{J}(n)$ the sign of the permutation induced on $J$ by conjugation with $n$. Then

$$
\sigma_{J}(n)=\epsilon(n) \alpha_{J}(n),
$$

for all $n \in N_{J}$.

Proof Denote by $V_{J}$ the orthogonal complement of Fix $\left(W_{J}\right)$ in $V$. Then $V_{J}$ affords the reflection representation of the parabolic subgroup $W_{J}$, and the decomposition $V=V_{J} \oplus \operatorname{Fix}\left(W_{J}\right)$ is $N_{W}\left(W_{J}\right)$-stable. For $n \in N_{J}$, the matrix of $n$ on $V_{J}$ is equivalent to the permutation matrix of the conjugation action of $n$ on $J$ and thus has determinant $\sigma_{J}(n)$. The matrix of $n$ on $\operatorname{Fix}\left(W_{J}\right)$ has determinant $\alpha_{J}(n)$, by definition. Consequently, the determinant of $n$ on $V$ is $\epsilon(n)=\sigma_{J}(n) \alpha_{J}(n)$.

Pfeiffer and Röhrle [13] call $W_{J}$ a bulky parabolic subgroup of $W$ if $N_{W}\left(W_{J}\right)$ is isomorphic to the direct product $W_{J} \times N_{J}$, or equivalently, if $N_{J}$ centralizes $W_{J}$. Notice that $W_{J}$ is bulky whenever $W_{J}$ is a self-normalizing subgroup of $W$. Suppose $W_{J}$ is bulky in $W$. Then $\sigma_{J}(n)=1$ for all $n \in N_{J}$. Consequently, for $u \in W_{J}$ and $n \in N_{J}$, we have

$$
\epsilon(u n) \alpha_{J}(u n)=\epsilon(u) .
$$

Thus, the character $\epsilon \alpha_{J}=\epsilon_{J} \times 1_{N_{J}}$ of $N_{W}\left(W_{J}\right)=W_{J} \times N_{J}$ is the trivial extension of the sign character of $W_{J}$.

Here and in the remainder of the paper, we denote the restrictions of the trivial and the sign character of $W$ to a subgroup $U$ of $W$ by $1_{U}$ and $\epsilon_{U}$, respectively, or by $1_{J}$ and $\epsilon_{J}$, if $U=W_{J}$ for some $J \subseteq S$. If no confusion can arise, we denote the restrictions of the characters $1_{S}$ and $\epsilon_{S}$ of $W$ to any of its subgroups simply by 1 and $\epsilon$, respectively.

Following Bergeron et al. [2], we decompose $\Sigma(W)$ into projective indecomposable modules, using a basis of quasi-idempotents, that naturally arise as follows. For $L, K \subseteq S$, we define

$$
m_{K L}= \begin{cases}\left|X_{K} \cap X_{L}^{\sharp}\right|, & \text { if } L \subseteq K, \\ 0, & \text { otherwise. }\end{cases}
$$

Then $\left(m_{K L}\right)_{K, L \subseteq S}$ is an invertible matrix, and consequently, there is a basis $\left(e_{L}\right)_{L \subseteq S}$ of $\Sigma(W)$ such that

$$
x_{K}=\sum_{L \subseteq S} m_{K L} e_{L}
$$

for $K \subseteq S$. Define, for $\lambda \in \Lambda$, elements

$$
e_{\lambda}=\sum_{L \in \lambda} e_{L}
$$

Then $\left\{e_{\lambda}: \lambda \in \Lambda\right\}$ is a set of primitive, pairwise orthogonal idempotents in $\Sigma(W)$. In particular,

$$
\sum_{\lambda \in \Lambda} e_{\lambda}=1 \in \mathbb{C} W
$$


Thus, if we set

$$
E_{\lambda}=e_{\lambda} \mathbb{C} W
$$

then

$$
\mathbb{C} W=\bigoplus_{\lambda \in \Lambda} E_{\lambda}
$$

is a decomposition of the group algebra into right ideals. We call the right ideal $E_{[S]}$ the top component of $\mathbb{C} W$.

For $\lambda \in \Lambda$, denote by $\Phi_{\lambda}$ the character of the $W$-module $E_{\lambda}$. Furthermore, for $L \subseteq S$, denote by $\Phi_{L}$ the character of the top component of the group algebra $\mathbb{C} W_{L}$. Notice that for $\lambda=[L], \Phi_{[L]}$ is a character of $W$ whereas $\Phi_{L}$ is a character of $W_{L}$. If $L=S$, then $W_{L}=W$ and $\Phi_{[S]}=\Phi_{S}$. In general, the characters $\Phi_{[L]}$ and $\Phi_{L}$ are related in the following way.

Proposition 2.4 [4, Corollary 3.13] Let $L \subseteq S$. Then the character $\Phi_{L}$ of $W_{L}$ extends to a character $\widetilde{\Phi}_{L}$ of the normalizer $N_{W}\left(W_{L}\right)=W_{L} \rtimes N_{L}$ such that

$$
\Phi_{[L]}=\operatorname{Ind}_{N_{W}\left(W_{L}\right)}^{W} \widetilde{\Phi}_{L}
$$

Remark 2.5 If $W_{L}$ is a bulky parabolic subgroup of $W$, then, by [4, Lemma 3.7] $\widetilde{\Phi}_{L}$ is the character $\Phi_{L} \times 1_{N_{L}}$ of $N_{W}\left(W_{L}\right)=W_{L} \times N_{L}$ and so $\Phi_{[L]}=\operatorname{Ind}_{W_{L} \times N_{L}}^{W}\left(\Phi_{L} \times\right.$ $\left.1_{N_{L}}\right)$.

\section{The reflection arrangement and the Orlik-Solomon algebra $A(W)$}

A finite Coxeter group of rank $r$ acts as a reflection group on Euclidean space $\mathbb{R}^{r}$. Here it is convenient to regard this as an action on the complex space $V_{\mathbb{C}}=\mathbb{C}^{r}$. Let

$$
T=\left\{s^{w}: s \in S, w \in W\right\}
$$

be the set of reflections of $W$. For $t \in T$, denote by $H_{t}$ the reflecting hyperplane of $t$, i.e., the 1-eigenspace of $t$. The set of hyperplanes $\mathcal{A}=\left\{H_{t}: t \in T\right\}$ is called the reflection arrangement of $W$; for details see [11, Chap. 6]. Examples of (the real part of) reflection arrangements in dimension 2 are shown in Figs. 1 and 2.

The lattice of $\mathcal{A}$ is the set of all possible intersections of hyperplanes

$$
L(\mathcal{A})=\left\{H_{t_{1}} \cap \cdots \cap H_{t_{p}}: t_{1}, \ldots, t_{p} \in T\right\} .
$$

For $X \in L(\mathcal{A})$, the pointwise stabilizer

$$
W_{X}=\{w \in W: x . w=x \text { for all } x \in X\}
$$

is a parabolic subgroup of $W$. We define the shape $\operatorname{sh}(X)$ of $X$ to be the shape of $W_{X}$, i.e., $\operatorname{sh}(X)=[L] \in \Lambda$ if $W_{X}$ is conjugate to $W_{L}$ in $W$ for some $L \subseteq S$. The group $W$ acts on $T$ by conjugation and the $W$-action on $T$ induces actions of $W$ on $\mathcal{A}$ and 

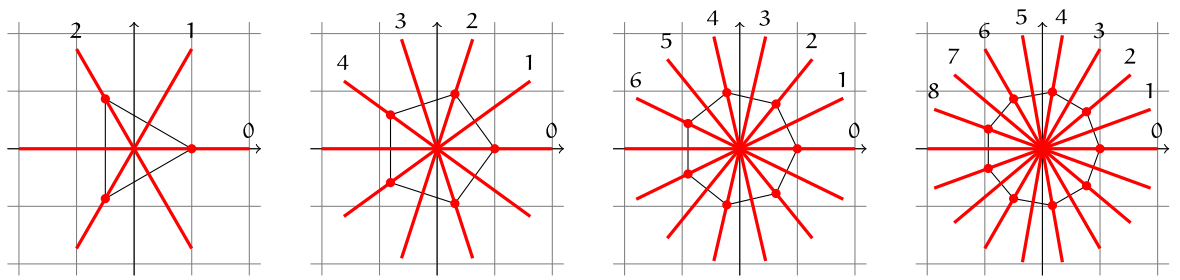

Fig. 1 Hyperplane arrangements of type $I_{2}(m), m=3,5,7,9$
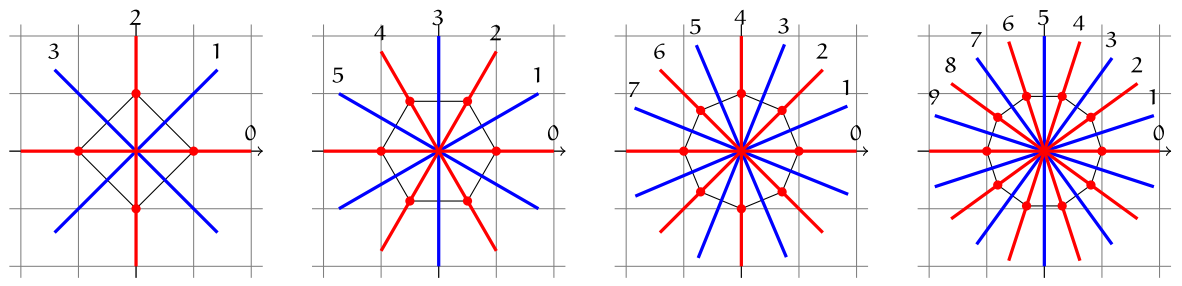

Fig. 2 Hyperplane arrangements of type $I_{2}(m), m=4,6,8,10$

$L(\mathcal{A})$. Orlik and Solomon [10] have shown that the normalizer of $W_{X}$ in $W$ is the setwise stabilizer of $X$ in $W$, that is,

$$
N_{W}\left(W_{X}\right)=\{w \in W: X . w=X\} .
$$

Consequently, the orbits of $W$ on the lattice $L(\mathcal{A})$ are parametrized by the shapes of $W$. We denote by $\alpha_{X}: N_{W}\left(W_{X}\right) \rightarrow \mathbb{C}$ the linear character of $N_{W}\left(W_{X}\right)$ defined by

$$
\alpha_{X}(w)=\operatorname{det}\left(\left.w\right|_{X}\right)
$$

for $w \in N_{W}\left(W_{X}\right)$. Then, for $w \in W$, we have $\alpha_{w}=\alpha_{X}$, where $X=\operatorname{Fix}(w)$, the fixed point subspace of $w$ in $V$. Moreover, for $L \subseteq S$, we have $\alpha_{L}=\alpha_{X}$, where $X=\operatorname{Fix}\left(W_{L}\right)$.

The Orlik-Solomon algebra of $W$ is the associative $\mathbb{C}$-algebra $A(W)$, generated as an algebra by elements $a_{t}, t \in T$, subject to the relations

$$
a_{t} a_{t^{\prime}}=-a_{t^{\prime}} a_{t}
$$

for all $t, t^{\prime} \in T$, and

$$
\sum_{i=1}^{p}(-1)^{i} a_{t_{1}} \cdots a_{t_{i-1}} \widehat{a_{t_{i}}} a_{t_{i+1}} \cdots a_{t_{p}}=0,
$$

where the hat denotes omission, whenever $\left\{H_{t_{1}}, \ldots, H_{t_{p}}\right\}$ is linearly dependent. The action of $W$ on the hyperplanes extends to an action on $A(W)$ via

$$
a_{t} \cdot w=a_{t} w
$$


for $t \in T, w \in W$. The algebra $A(W)$ is a skew-commutative, graded algebra

$$
A(W)=\bigoplus_{p \geq 0} A^{p},
$$

where the degree $p$ subspace $A^{p}$ is spanned by those monomials $a_{t_{1}} \cdots a_{t_{p}}$ in $A(W)$ with $\operatorname{dim} H_{t_{1}} \cap \ldots \cap H_{t_{p}}=r-p$. Clearly, $A^{p}=0$ for $p>r$. We call $A^{r}$ the top component of $A(W)$. We need a refinement of this decomposition, due to Brieskorn [3]. For a subspace $X \in L(\mathcal{A})$ of codimension $p$, define a subspace

$$
A_{X}=\left\langle a_{t_{1}} \cdots a_{t_{p}}: H_{t_{1}} \cap \ldots \cap H_{t_{p}}=X\right\rangle
$$

of $A(W)$. Then $A_{\{0\}}=A^{r}$ is the top component of $A(W)$. Note that $A_{X}$ is an embedding of the top component of $A\left(W_{X}\right)$ into $A(W)$. For $w \in W$, we have $A_{X} \cdot w=A_{X . w}$ and so $A_{X}$ is an $N_{W}\left(W_{X}\right)$-stable subspace.

We have

$$
A(W)=\bigoplus_{X \in L(\mathcal{A})} A_{X}
$$

and if we set

$$
A_{\lambda}=\bigoplus_{\operatorname{sh}(X)=\lambda} A_{X},
$$

for $\lambda \in \Lambda$, then

$$
A(W)=\bigoplus_{\lambda \in \Lambda} A_{\lambda}
$$

is a decomposition of $A(W)$ into $W$-modules $A_{\lambda}$. Note that $A_{[S]}=A_{\{0\}}$ is the top component of $A(W)$.

For $\lambda \in \Lambda$, denote by $\Psi_{\lambda}$ the character of the component $A_{\lambda}$ of the Orlik-Solomon algebra $A(W)$. Furthermore, for $L \subseteq S$, denote by $\Psi_{L}$ the character of the top component of the Orlik-Solomon algebra $A\left(W_{L}\right)$ of the parabolic subgroup $W_{L}$ of $W$. Notice that for $\lambda=[L], \Psi_{[L]}$ is a character of $W$ whereas $\Psi_{L}$ is a character of $W_{L}$. If $L=S$, then $\Psi_{[S]}=\Psi_{S}$. In general, the characters $\Psi_{[L]}$ and $\Psi_{L}$ are related in the following way, analogous to Proposition 2.4.

Proposition $3.1[9, \S 2]$ Let $L \subseteq S$. Then the character $\Psi_{L}$ of $W_{L}$ extends to a character $\widetilde{\Psi}_{L}$ of the normalizer $N_{W}\left(W_{L}\right)=W_{L} \rtimes N_{L}$ such that

$$
\Psi_{[L]}=\operatorname{Ind}_{N_{W}\left(W_{L}\right)}^{W} \widetilde{\Psi}_{L}
$$

Remark 3.2 Suppose that $W_{L}$ is a bulky parabolic subgroup of $W$ and set $X=$ Fix $\left(W_{L}\right)$. If codim $X=p$ and $t_{1}, \ldots, t_{p}$ are in $T$ with $X=H_{t_{1}} \cap \cdots \cap H_{t_{p}}$, then $t_{1}, \ldots, t_{p}$ are in $W_{L}$ and so, since $N_{L}$ centralizes $W_{L}$, we have $a_{t_{1}} \cdots a_{t_{p}} . n=$ $a_{t_{1}^{n}} \cdots a_{t_{p}^{n}}=a_{t_{1}} \cdots a_{t_{p}}$, for $n \in N_{L}$. Thus, $\widetilde{\Psi}_{L}$ is the character $\Psi_{L} \times 1_{N_{L}}$ of $N_{W}\left(W_{L}\right)=$ $W_{L} \times N_{L}$ and so $\Psi_{[L]}=\operatorname{Ind}_{W_{L} \times N_{L}}^{W}\left(\Psi_{L} \times 1_{N_{L}}\right)$. 


\section{The inductive strategy}

Before stating our relative Conjecture $\mathrm{C}$, we briefly review the proof of Conjecture 2.1 in [4] and describe how it leads to a proof of Conjecture A. We first showed that the characters of the top components of $\mathbb{C} W$ and $A(W)$ are related as described in the following conjecture which makes sense for any finite Coxeter group. To this end, let $\mathcal{C}$ be the set of cuspidal conjugacy classes of $W$ and, for $L \subseteq S$, let $\mathcal{C}_{L}$ denote the set of cuspidal conjugacy classes in $W_{L}$. For a class $C$ in $\mathcal{C}$ or $\mathcal{C}_{L}$, we denote by $w_{C} \in C$ a fixed representative.

Conjecture B For each class $C \in \mathcal{C}$, there exist linear characters $\varphi_{w_{C}}$ and $\psi_{w_{C}}$ of the centralizer $C_{W}\left(w_{C}\right)$ such that the following hold:

(i) $\Phi_{S}=\sum_{C \in \mathcal{C}} \operatorname{Ind}_{C_{W}\left(w_{C}\right)}^{W} \varphi_{w_{C}}$;

(ii) $\Psi_{S}=\sum_{C \in \mathcal{C}} \operatorname{Ind}_{C_{W}\left(w_{C}\right)}^{W} \psi_{w_{C}}$;

(iii) $\psi_{w_{C}}=\varphi_{w_{C}} \in$ for all $C \in \mathcal{C}$.

Remark 4.1 If it is known that $\Psi_{S}=\Phi_{S} \epsilon_{S}$, then choosing $\psi_{w_{C}}$ or $\varphi_{w_{C}}$ in such a way that $\psi_{w_{C}}=\varphi_{w_{C}} \epsilon$, we have that part (iii) in the above Conjecture B holds and that $(i)$ and (ii) are equivalent statements.

When $W$ is a symmetric group, every parabolic subgroup $W_{L}$ of $W$ is a product of symmetric groups and so Conjecture B holds for the group $W_{L}$. Thus, for $w_{C} \in C \in$ $\mathcal{C}_{L}$, we obtained linear characters $\varphi_{w_{C}}$ and $\psi_{w_{C}}$ of $C_{W_{L}}\left(w_{C}\right)$ such that the characters $\Phi_{L}$ and $\Psi_{L}$ of $W_{L}$ decompose as

$$
\Phi_{L}=\sum_{C \in \mathcal{C}_{L}} \operatorname{Ind}_{C_{W_{L}}\left(w_{C}\right)}^{W_{L}} \varphi_{w_{C}} \quad \text { and } \quad \Psi_{L}=\sum_{C \in \mathcal{C}_{L}} \operatorname{Ind}_{C_{W_{L}}\left(w_{C}\right)}^{W_{L}} \psi_{w_{C}} .
$$

We know from Propositions 2.4 and 3.1 that $\Phi_{L}$ and $\Psi_{L}$ extend to characters $\widetilde{\Phi}_{L}$ and $\widetilde{\Psi}_{L}$ of $N_{W}\left(W_{L}\right)$. The next step in [4] was to show that each $\varphi_{w_{C}}$ and $\psi_{w_{C}}$ extend to characters $\widetilde{\varphi}_{w_{C}}$ and $\widetilde{\psi}_{w_{C}}$ of $C_{W}\left(w_{C}\right)$ in such a way that

$$
\widetilde{\Phi}_{L}=\sum_{C \in \mathcal{C}_{L}} \operatorname{Ind}_{C_{W}\left(w_{C}\right)}^{N_{W}\left(W_{L}\right)} \tilde{\varphi}_{w_{C}} \quad \text { and } \quad \widetilde{\Psi}_{L}=\sum_{C \in \mathcal{C}_{L}} \operatorname{Ind}_{C_{W}\left(w_{C}\right)}^{N_{W}\left(W_{L}\right)} \widetilde{\psi}_{w_{C}},
$$

and moreover that $\widetilde{\psi}_{w_{C}}=\widetilde{\varphi}_{w_{C}} \epsilon \alpha_{L}$ for all $C \in \mathcal{C}_{L}$. Finally, we applied $\operatorname{Ind}_{N_{W}\left(W_{L}\right)}^{W}$ to (4.2) and summed over the set of shapes $[L] \in \Lambda$. Conjecture $A$ then followed immediately by transitivity of induction.

Motivated by (4.2), we make the following general conjecture.

Conjecture $\mathbf{C}$ Let $L \subseteq S$. Then, for each $C \in \mathcal{C}_{L}$, there exist linear characters $\widetilde{\varphi}_{w_{C}}$ and $\widetilde{\psi}_{w_{C}}$ of $C_{W}\left(w_{C}\right)$ such that the following hold:

(i) $\widetilde{\Phi}_{L}=\sum_{C \in \mathcal{C}_{L}} \operatorname{Ind}_{C_{W}\left(w_{C}\right)}^{N_{W}\left(W_{L}\right)} \widetilde{\varphi}_{w_{C}}$;

(ii) $\widetilde{\Psi}_{L}=\sum_{C \in \mathcal{C}_{L}} \operatorname{Ind}_{C_{W}\left(w_{C}\right)}^{N_{W}\left(W_{L}\right)} \widetilde{\psi}_{w_{C}}$;

(iii) $\widetilde{\psi}_{w_{C}}=\widetilde{\varphi}_{w_{C}} \epsilon_{S} \alpha_{L}$ for all $C \in \mathcal{C}_{L}$. 
Remark 4.3 If it is known that $\widetilde{\Psi}_{L}=\widetilde{\Phi}_{L} \epsilon_{S} \alpha_{L}$, then choosing $\widetilde{\psi}_{w_{C}}$ or $\widetilde{\varphi}_{w_{C}}$ in such a way that $\widetilde{\psi}_{w_{C}}=\widetilde{\varphi}_{w_{C}} \epsilon_{S} \alpha_{L}$, we have that part (iii) in the above Conjecture C holds and that $(i)$ and (ii) are equivalent statements.

Conjecture $\mathrm{B}$ is known to hold in the following cases:

1. $W$ of type $A$ (see [4, Theorem 4.1]);

2. $W$ has rank 2 or less (see Lemmas 5.1 and 5.2, Theorem 5.11).

Conjecture $\mathrm{C}$ is known to hold in the following cases:

1. $W$ of type $A$; all $L$ (see [4, Theorem 5.2]);

2. $W$ arbitrary; $W_{L}$ is bulky and satisfies Conjecture B (by Theorem 4.7);

3. $W$ arbitrary; $|L| \leq 2$ (see Corollary 5.3, Theorem 5.18).

If Conjecture $\mathrm{C}$ holds for all $L \subseteq S$, then Conjecture A is true for $W$.

Theorem 4.4 Suppose that Conjecture C holds for all subsets $L \subseteq S$. Then for each $w$ in a set $\mathcal{R}$ of representatives of the conjugacy classes of $W$, there are linear characters $\widetilde{\varphi}_{w}$ and $\widetilde{\psi}_{w}$ of $C_{W}(w)$ such that

(i) the regular character of $W$ is given by $\rho_{W}=\sum_{w \in \mathcal{R}} \operatorname{Ind}_{C_{W}(w)}^{W} \tilde{\varphi}_{w}$,

(ii) the Orlik-Solomon character of $W$ is given by $\omega_{W}=\sum_{w \in \mathcal{R}} \operatorname{Ind}_{C_{W}(w)}^{W} \widetilde{\psi}_{w}$, and

(iii) $\widetilde{\psi}_{w}=\widetilde{\varphi}_{w} \epsilon \alpha_{w}$ for all $w \in \mathcal{R}$.

Proof For $L \subseteq S$, let $\mathcal{R}_{L}$ be a set of representatives of the classes $\mathcal{C}_{L}$. For a class $C \in \mathcal{C}_{L}$, denote by $w_{C} \in \mathcal{R}_{L}$ its representative. Let $\mathcal{L}$ be a set of representatives of shapes, so $\Lambda=\{[L] \mid L \in \mathcal{L}\}$. Then, by [5, Theorem 3.2.12], we may assume without loss that

$$
\mathcal{R}=\coprod_{L \in \mathcal{L}} \mathcal{R}_{L}=\left\{w_{C}: C \in \mathcal{C}_{L}, L \in \mathcal{L}\right\}
$$

Then, by Conjecture $\mathrm{C}$ the equality in (iii) holds. By (2.3) and Proposition 2.4, we have

$$
\rho_{W}=\sum_{\lambda \in \Lambda} \Phi_{\lambda}=\sum_{L \in \mathcal{L}} \operatorname{Ind}_{N_{W}\left(W_{L}\right)}^{W} \widetilde{\Phi}_{L}=\sum_{L \in \mathcal{L}} \sum_{C \in \mathcal{C}_{L}} \operatorname{Ind}_{C_{W}\left(w_{C}\right)}^{W} \tilde{\varphi}_{w_{C}},
$$

as desired. The formula for $\omega_{W}$ follows in the same way.

Notice that in the case when $L=S$, Conjecture $\mathrm{C}$ is simply a restatement of Conjecture B. In general, Conjecture $\mathrm{C}$ for $L \subseteq S$ implies the validity of Conjecture B for the group $W_{L}$, as follows.

Proposition 4.5 Suppose that Conjecture C holds for a subset $L \subseteq S$. Then the restrictions

$$
\varphi_{w_{C}}=\operatorname{Res}_{C_{W_{L}}\left(w_{C}\right)}^{C_{W}\left(w_{C}\right)} \tilde{\varphi}_{w_{C}} \quad \text { and } \quad \psi_{w_{C}}=\operatorname{Res}_{C_{W_{L}}\left(w_{C}\right)}^{C_{W}\left(w_{C}\right)} \widetilde{\psi}_{w_{C}}
$$

are linear characters that satisfy Conjecture B for $W_{L}$. 
Proof By Mackey's theorem, we have

$$
\operatorname{Res}_{W_{L}}^{N_{W}\left(W_{L}\right)} \operatorname{Ind}_{C_{W}\left(w_{C}\right)}^{N_{W}\left(W_{L}\right)} \tilde{\varphi}_{w_{C}}=\operatorname{Ind}_{C_{W_{L}}\left(w_{C}\right)}^{W_{L}} \operatorname{Res}_{C_{W_{L}}\left(w_{C}\right)}^{C_{W}\left(w_{C}\right)} \tilde{\varphi}_{w_{C}},
$$

since $N_{W}\left(W_{L}\right)=W_{L} C_{W}\left(w_{C}\right)$ (see [8]), and therefore,

$$
\begin{aligned}
\Phi_{L} & =\operatorname{Res}_{W_{L}}^{N_{W}\left(W_{L}\right)} \widetilde{\Phi}_{L} \\
& =\sum_{C \in \mathcal{C}_{L}} \operatorname{Res}_{W_{L}}^{N_{W}\left(W_{L}\right)} \operatorname{Ind}_{C_{W}\left(w_{C}\right)}^{N_{W}\left(W_{L}\right)} \widetilde{\varphi}_{w_{C}} \\
& =\sum_{C \in \mathcal{C}_{L}} \operatorname{Ind}_{C_{W_{L}}\left(w_{C}\right)}^{W_{L}} \operatorname{Res}_{C_{W_{L}}\left(w_{C}\right)}^{C_{W}\left(w_{C}\right)} \widetilde{\varphi}_{w_{C}} \\
& =\sum_{C \in \mathcal{C}_{L}} \operatorname{Ind}_{C_{W_{L}}\left(w_{C}\right)}^{W_{L}} \varphi_{w_{C}} .
\end{aligned}
$$

The formula for $\Psi_{L}$ follows in the same way. The conclusion that $\psi_{w_{C}}=\varphi_{w_{C}} \epsilon$ for $C \in \mathcal{C}_{L}$ is easily seen to hold.

Remark 4.6 Although Conjecture B for $W_{L}$ formally follows from Conjecture $C$, as in [4], the characters $\widetilde{\varphi}_{w_{C}}$ and $\widetilde{\psi}_{w_{C}}$ of $C_{W}\left(w_{C}\right)$ arise in practice as extensions of characters $\varphi_{w_{C}}$ and $\psi_{w_{C}}$ of $C_{W_{L}}\left(w_{c}\right)$ that satisfy Conjecture B for $W_{L}$. In particular, if Conjecture $B$ is known to hold for $W_{L}$, then using Remark 4.3, to prove Conjecture $C$ for $L \subseteq S$, it suffices to prove that each $\varphi_{w_{C}}$ extends to $C_{W}\left(w_{C}\right)$ in such a way that Conjecture $C(i)$ holds and that $\widetilde{\Psi}_{L}=\widetilde{\Phi}_{L} \epsilon_{S} \alpha_{L}$.

When $L \subseteq S$ is such that $W_{L}$ is a self-normalizing subgroup of $W$ (e.g., if $L=S$ ), then $N_{L}$ is the trivial group and Conjecture B for the group $W_{L}$ vacuously implies Conjecture $\mathrm{C}$ for the subset $L$ in this case. More generally, whenever the complement $N_{L}$ centralizes $W_{L}$, i.e., when $W_{L}$ is bulky in $W$, Conjecture B for $W_{L}$ implies Conjecture $\mathrm{C}$ for $L \subseteq S$, as follows.

Theorem 4.7 Let $L \subseteq S$. Suppose that Conjecture B holds for the group $W_{L}$ and that $W_{L}$ is a bulky parabolic subgroup of $W$. Then Conjecture C holds with $\widetilde{\varphi}_{w_{C}}=$ $\varphi_{w_{C}} \times 1_{N_{L}}$ and $\widetilde{\psi}_{w_{C}}=\psi_{w_{C}} \times 1_{N_{L}}$ for each cuspidal class $C$ of $W_{L}$.

Proof As observed in the remark above, it suffices to show that each $\varphi_{w_{C}}$ extends to $C_{W}\left(w_{C}\right)$ in such a way that Conjecture C (i) holds and that $\widetilde{\Psi}_{L}=\widetilde{\Phi}_{L} \epsilon_{S} \alpha_{L}$.

Because $N_{L}$ centralizes $W_{L}$, we have that the centralizer $C_{W}\left(w_{C}\right)$ is the direct product of $C_{W_{L}}\left(w_{C}\right)$, and $N_{L}$ and so $\widetilde{\varphi}_{w_{C}}$ is indeed a linear character of $C_{W}\left(w_{C}\right)$ that extends $\varphi_{w_{C}}$. Thanks to Remark 2.5, $\widetilde{\Phi}_{L}=\Phi_{L} \times 1_{N_{L}}$. Thus, by Conjecture B (i), we have 


$$
\begin{aligned}
\widetilde{\Phi}_{L} & =\Phi_{L} \times 1_{N_{L}}=\left(\sum_{C \in \mathcal{C}_{L}} \operatorname{Ind}_{C_{W_{L}}\left(w_{C}\right)}^{W_{L}} \varphi_{w_{C}}\right) \times 1_{N_{L}} \\
& =\sum_{C \in \mathcal{C}_{L}} \operatorname{Ind}_{C_{W_{L}}\left(w_{C}\right) \times N_{L}}^{W_{L} \times N_{L}}\left(\varphi_{w_{C}} \times 1_{N_{L}}\right) \\
& =\sum_{C \in \mathcal{C}_{L}} \operatorname{Ind}_{C_{W}\left(w_{C}\right)}^{N_{W}\left(W_{L}\right)} \widetilde{\varphi}_{w_{C}} .
\end{aligned}
$$

Hence Conjecture C (i) holds.

By Remark 3.2, Conjecture B (iii), Lemma 2.1, and Remark 2.5, we have

$$
\widetilde{\Psi}_{L}=\Psi_{L} \times 1_{N_{L}}=\Phi_{L} \epsilon_{L} \times 1_{N_{L}} \sigma_{L}=\left(\Phi_{L} \times 1_{N_{L}}\right) \epsilon \alpha_{L}=\widetilde{\Phi}_{L} \epsilon_{S} \alpha_{L}
$$

using the fact that $W_{L} \subseteq \operatorname{ker} \alpha_{L}$, whence we are done.

Combining Theorem 4.7 with the results in [4], we see that if $W_{L}$ is a product of Coxeter groups of type $A$ and is a bulky parabolic subgroup of $W$, then Conjecture $C$ holds for $L \subseteq S$. For example, if $W_{L}$ is of type $A_{1} \times A_{3}$ and $W$ is of type $E_{6}$, then the characters $\varphi_{w_{C}}$ and $\psi_{w_{C}}$ constructed in [4] satisfy Conjecture B and so, by Theorem 4.7, they extend to $C_{W}\left(w_{C}\right)$ and Conjecture $\mathrm{C}$ holds. Note, however, that the property of being a bulky parabolic subgroup depends in a fundamental way on the embedding of $W_{L}$ in $W$. If $W_{L}$ is of type $A_{1} \times A_{3}$ and $W$ is of type $E_{7}$, then $W_{L}$ is not bulky and Theorem 4.7 cannot be applied.

\section{Conjectures A, B and C for Coxeter groups of rank up to 2}

In this section, we show that Conjecture $\mathrm{C}$ holds for $L \subseteq S$ for any $S$ as long as $|L| \leq 2$. Note that because the type of the ambient Coxeter group $W$ is arbitrary, even for types $A_{1} \times A_{1}$ and $A_{2}$ Conjecture $\mathrm{C}$ is a stronger statement than is proved in [4] for such parabolic subgroups. The strategy we use is to first prove that Conjecture B holds for $W$ when the rank of $W$ is at most 2 and then use the procedure outlined in Remark 4.6. Combining Conjecture $\mathrm{C}$ with Theorem 4.7, we conclude that Conjectures $\mathrm{A}, \mathrm{B}$, and $\mathrm{C}$ all hold in case the rank of $W$ is at most 2.

The top components of Coxeter groups of rank 0 or 1 almost trivially satisfy Conjecture B. For later reference, we record this explicitly in the following lemmas.

Lemma 5.1 The top component characters of $W_{\varnothing}$ are $\Phi_{\varnothing}=1_{\varnothing}$ and $\Psi_{\varnothing}=1_{\varnothing}$. Moreover, $W_{\varnothing}$ satisfies Conjecture $B$ with $\varphi_{1}=1_{\varnothing}$ and $\psi_{1}=1_{\varnothing}$.

Lemma 5.2 Suppose $W$ is a Coxeter group of rank 1 , generated by $S=\{s\}$. Then the top component characters of $W$ are $\Phi_{S}=\epsilon_{S}$ and $\Psi_{S}=1_{S}$. Moreover, $W$ satisfies Conjecture $B$ with $\varphi_{s}=\epsilon_{S}$ and $\psi_{s}=1_{S}$.

Proof In this case, the non-trivial conjugacy class $\{s\}$ is the unique cuspidal conjugacy class in $W$. From the definitions we have $e_{[S]}=e_{S}=\frac{1}{2}(1-s)$ and it follows that 
$W$ acts on the top component $E_{[S]}=e_{[S]} \mathbb{C} W$ with character $\Phi_{[S]}=\epsilon_{S}$. Moreover, $W$ acts trivially on the basis $\left\{a_{S}\right\}$ of the top component $A_{[S]}$ of $A(W)$, which therefore affords the trivial character. Thus, $\Psi_{[S]}=1_{S}$ and so $\Phi_{[S]}=\Psi_{[S]} \epsilon_{S}$. Set $\varphi_{S}=\epsilon_{S}$ and $\psi_{s}=1_{S}$. Then $\varphi_{s}$ and $\psi_{s}$ obviously satisfy the conclusions of Conjecture B.

In any finite Coxeter group $W$, parabolic subgroups of rank 0 and 1 are always bulky. We may thus conclude from Lemmas 5.1 and 5.2 and Theorem 4.7 that Conjecture C holds for $L \subseteq S$ with $|L| \leq 1$.

Corollary 5.3 Suppose that $L \subseteq S$ has size $|L| \leq 1$. Then Conjecture C holds.

As a consequence of the corollary, $W$ acts trivially on both the component $E_{[\varnothing]}$ of the group algebra $\mathbb{C} W$ (with character $\Phi_{[\varnothing]}=\widetilde{\Phi}_{\varnothing}=1_{S}$ ) and the component $A_{[\varnothing]}$ of the Orlik-Solomon algebra $A(W)$ (with character $\Psi_{[\varnothing]}=\widetilde{\Psi}_{\varnothing}=1_{S}$ ), as one can easily establish directly.

Moreover, the degree 1 component of $A(W)$ is a direct sum of transitive permutation modules, one for each conjugacy class of reflections of $W$. This agrees with the description of the degree 1 component of $A(W)$ as the permutation representation of $W$ on its reflections, that can easily be obtained directly.

Next we consider the case when $W$ has rank 2 . Until further notice, we assume that

$$
W=\left\langle s, t: s^{2}=t^{2}=(s t)^{m}=1\right\rangle .
$$

Then $W$ is a Coxeter group of rank two and is of type $A_{1} \times A_{1}$, or $I_{2}(m)$ for $m \geq 3$, with Coxeter generators $S=\{s, t\}$. For convenience, we regard type $A_{1} \times A_{1}$ as type $I_{2}(2)$, noting that the general results of this section remain true for $m=2$.

To prove Conjecture B for $W$, we first compute the character $\Phi_{S}$ of the top component $E_{[S]}$ of the group algebra $\mathbb{C} W$, and verify that it is a sum of induced linear characters. Then we compute the character $\Psi_{S}$ of the top component $A_{[S]}$ of the Orlik-Solomon algebra $A(W)$ and verify that $\Psi_{S}=\Phi_{S} \epsilon_{S}$. Conjecture B then follows as observed in Remark 4.1.

As usual, denote by $w_{0}$ the longest element of $W$. Furthermore, we define

$$
\operatorname{Av}(U)=\frac{1}{|U|} \sum_{u \in U} u
$$

for a subgroup $U$ of $W$. Recall that $\operatorname{Av}(U) u=\operatorname{Av}(U)$ for all $u \in U$ and that $\operatorname{Av}(U) \mathbb{C} W$ is the permutation module of $W$ on the cosets of $U$.

Lemma $5.4 e_{S}=\operatorname{Av}\left(\left\langle w_{0}\right\rangle\right)-\operatorname{Av}(W)$.

Proof By Solomon's theorem [15], the elements

$$
\begin{array}{ll}
x_{\varnothing}=1+s+t+s t+t s+\cdots+w_{0}, & x_{s}=1+t+s t+t s t+\cdots+w_{0} s, \\
x_{s t}=1, & x_{t}=1+s+t s+s t s+\cdots+w_{0} t
\end{array}
$$

form a basis of the descent algebra $\Sigma(W)$. Note that $x_{t}+x_{s}=x_{\varnothing}+1-w_{0}$. 
For $L \subseteq K \subseteq S$, the numbers $m_{K L}=\left|X_{K} \cap X_{L}^{\sharp}\right|$ are easily determined as

$$
\left(m_{K L}\right)_{K, L \subseteq S}=\left[\begin{array}{cccc}
2 m & \cdot & \cdot & \cdot \\
m & 2 & \cdot & \cdot \\
m & . & 2 & . \\
1 & 1 & 1 & 1
\end{array}\right], \quad\left(m_{K L}\right)^{-1}=\left[\begin{array}{cccc}
\frac{1}{2 m} & \cdot & \cdot & \cdot \\
-\frac{1}{4} & \frac{1}{2} & \cdot & \cdot \\
-\frac{1}{4} & \cdot & \frac{1}{2} & \cdot \\
\frac{m-1}{2 m} & -\frac{1}{2} & -\frac{1}{2} & 1
\end{array}\right] .
$$

Hence the idempotents $e_{L}$ are (cf. [2])

$$
\begin{aligned}
e_{\varnothing} & =\frac{1}{2 m} x_{\varnothing}, & e_{s} & =\frac{1}{2} x_{s}-\frac{1}{4} x_{\varnothing}, \\
e_{s t} & =1-\frac{1}{2} x_{s}-\frac{1}{2} x_{t}+\frac{m-1}{2 m} x_{\varnothing}, & e_{t} & =\frac{1}{2} x_{t}-\frac{1}{4} x_{\varnothing} .
\end{aligned}
$$

From $x_{t}+x_{s}=1+x_{\varnothing}-w_{0}$, it follows that $e_{s}+e_{t}=\frac{1}{2}\left(1-w_{0}\right)$, and hence that $e_{S}=\frac{1}{2}\left(1+w_{0}\right)-e_{\varnothing}=\operatorname{Av}\left(\left\langle w_{0}\right\rangle\right)-\operatorname{Av}(W)$, as required.

As an immediate consequence we obtain the character of the top component of $\mathbb{C} W$.

Corollary 5.5 The $W$-module $E_{[S]}$ affords the character $\Phi_{S}=\operatorname{Ind}_{\left\langle w_{0}\right\rangle}^{W}(1)-1_{S}$.

Next we identify linear characters of centralizers of cuspidal elements. Note that the group $W$ consists of $m$ reflections and $m$ rotations. Denote the rotation subgroup of $W$ by $W^{+}=\langle s t\rangle$. The centralizer of a rotation $w$ is $W^{+}$, unless $w$ is central in $W$. The cuspidal classes of $W$ are exactly the classes of nontrivial rotations, represented by the set $\left\{(s t)^{j}: j=1, \ldots,\left\lfloor\frac{m}{2}\right\rfloor\right\}$, containing $w_{0}=(s t)^{m / 2}$ in case $m$ is even. The group $W^{+}$is a cyclic group of order $m$ and it has $m$ linear characters $\chi_{j}$, $j=0, \ldots, m-1$, defined by

$$
\chi_{j}(s t)=\zeta_{m}^{j}
$$

for a primitive $m$ th root of unity $\zeta_{m}$. In the following arguments, we make frequent use of the fact that the sum of all the nontrivial characters $\chi_{j}$ of $W^{+}$equals the difference of its regular and its trivial character,

$$
\sum_{j=1}^{m-1} \chi_{j}=\operatorname{Ind}_{\{1\}}^{W^{+}}(1)-1_{W^{+}},
$$

which obviously follows from $\sum_{j=0}^{m-1} \chi_{j}=\operatorname{Ind}_{\{1\}}^{W^{+}}(1)$ and $\chi_{0}=1_{W^{+}}$.

We distinguish two cases, depending on the parity of $m$.

Proposition 5.6 Suppose that $m=2 k$ with $k>0$. Let

$$
\varphi_{(s t)^{j}}= \begin{cases}\chi_{2 j}, & 0<j<k, \\ \epsilon_{S}, & j=k .\end{cases}
$$


Then $\varphi_{(s t)^{j}}$ is a linear character of $C_{W}\left((s t)^{j}\right)$, for $j=1, \ldots, k$, and

$$
\sum_{j=1}^{k} \operatorname{Ind}_{C_{W}\left((s t)^{j}\right)}^{W}\left(\varphi_{(s t)^{j}}\right)=\epsilon_{S}+\sum_{j=1}^{k-1} \operatorname{Ind}_{W^{+}}^{W}\left(\chi_{2 j}\right)=\Phi_{S}
$$

Proof Note that $C_{W}\left((s t)^{j}\right)=W^{+}$and $w_{0}$ lies in the kernel of the characters $\varphi_{(s t)^{j}}=$ $\chi_{2 j}$, for all $j=1, \ldots, k-1$. Hence the $\chi_{2 j}$ can be regarded as a full set of nontrivial irreducible characters of the quotient group $W^{+} /\left\langle w_{0}\right\rangle$, whence their sum $\sum_{j=1}^{k-1} \chi_{2 j}$ equals the difference of its regular and its trivial characters. Thus, as a character of $W^{+}$, we have

$$
\sum_{j=1}^{k-1} \chi_{2 j}=\operatorname{Ind}_{\left\langle w_{0}\right\rangle}^{W^{+}}(1)-1_{W^{+}}
$$

Therefore

$$
\epsilon_{S}+\operatorname{Ind}_{W^{+}}^{W}\left(\sum_{j=1}^{k-1} \chi_{2 j}\right)=\epsilon_{S}+\operatorname{Ind}_{\left\langle w_{0}\right\rangle}^{W}(1)-\operatorname{Ind}_{W^{+}}^{W}(1)=\operatorname{Ind}_{\left\langle w_{0}\right\rangle}^{W}(1)-1_{S}=\Phi_{S}
$$

where the penultimate equality holds because $\operatorname{Ind}_{W^{+}}^{W}(1)=1_{S}+\epsilon_{S}$.

Proposition 5.7 Suppose that $m=2 k+1$ for some $k>0$. For $j=1, \ldots, k$, let

$$
\varphi_{(s t)^{j}}=\chi_{j}
$$

Then $\varphi_{(s t)^{j}}$ is a linear character of $C_{W}\left((s t)^{j}\right)$, for $j=1, \ldots, k$, and

$$
\sum_{j=1}^{k} \operatorname{Ind}_{C_{W}\left((s t)^{j}\right)^{j}}^{W}\left(\varphi_{(s t)^{j}}\right)=\sum_{j=1}^{k} \operatorname{Ind}_{W^{+}}^{W}\left(\chi_{j}\right)=\Phi_{S}
$$

Proof We have $C_{W}\left((s t)^{j}\right)=W^{+}$and $\operatorname{Res}_{W^{+}}^{W}\left(\operatorname{Ind}_{W^{+}}^{W}\left(\chi_{j}\right)\right)=\chi_{j}+\chi_{m-j}$ for all $j=$ $1, \ldots, k$. Hence

$$
\begin{aligned}
\operatorname{Res}_{W^{+}}^{W}\left(\sum_{j=1}^{k} \operatorname{Ind}_{W^{+}}^{W}\left(\chi_{j}\right)\right) & =\sum_{j=1}^{m-1} \chi_{j}=\operatorname{Ind}_{\{1\}}^{W^{+}}(1)-1_{W^{+}} \\
& =\operatorname{Res}_{W^{+}}^{W}\left(\operatorname{Ind}_{\left\langle w_{0}\right\rangle}^{W}(1)-1_{S}\right)=\operatorname{Res}_{W^{+}}^{W}\left(\Phi_{S}\right) .
\end{aligned}
$$

It follows that

$$
\Phi_{S}=\sum_{j=1}^{k} \operatorname{Ind}_{W^{+}}^{W}\left(\chi_{j}\right)
$$

since the restrictions of both characters to the subgroup $\left\langle w_{0}\right\rangle$ of $W$ also coincide. 
Proposition 5.8 Let $\pi_{\mathcal{A}}$ be the character of the permutation action of $W$ on the hyperplane arrangement $\mathcal{A}$. Then $W$ acts on the degree 1 component of $A(W)$ with character $\pi_{\mathcal{A}}$, and $W$ acts on the component $A_{[S]}$ of $A(W)$ with character

$$
\Psi_{S}=\pi_{\mathcal{A}}-1_{S} .
$$

Consequently, $W$ acts on $A(W)$ with character $2 \pi_{\mathcal{A}}$.

Proof The degree 1 component of $A(W)$ has basis $\left\{a_{t}: t \in T\right\}$ and $W$ acts on it by permuting the basis vectors. In order to analyze the top component of $A(W)$, we make this permutation action explicit as follows.

Label the hyperplanes $H_{0}, \ldots, H_{m-1}$, so that the real part of the hyperplane $H_{j}$ is spanned by $\zeta_{2 m}^{j}$, where $\zeta_{2 m}=e^{2 \pi i / 2 m}$ is a primitive $(2 m)$ th root of unity, as shown in Figs. 1 and 2.

Let $s$ be the reflection about $H_{0}$ (the $x$-axis) and $t s=(s t)^{-1}$ the (anti-clockwise) rotation about the angle $2 \pi / m$. Then $t$ is the reflection about $H_{m-1}$.

The reflection $s$ permutes the hyperplanes according to the rule

$$
H_{j} . s=H_{m-j},
$$

for $j=0, \ldots, m-1$, fixing $H_{0}$. The rotation $t s$ acts as

$$
H_{j} . t s=H_{j+2} \text {, }
$$

for $j=0, \ldots, m-1$, where the indices are reduced $\bmod m$ if necessary.

The top component $A_{[S]}$ has a basis $\left\{a_{0} a_{j}: j=1, \ldots, m-1\right\}$, where $W$ acts on the indices as indicated above, subject to the relation $a_{0} a_{j}-a_{0} a_{k}+a_{j} a_{k}=0$, i.e.,

$$
a_{j} a_{k}=a_{0} a_{k}-a_{0} a_{j}
$$

The reflection $s$ fixes $H_{0}$ and thus maps $a_{0} a_{j}$ to

$$
a_{0} a_{j} . s=a_{0} a_{m-j},
$$

for $j=1, \ldots, m-1$. The rotation $t s$ maps $a_{0} a_{j}$ to

$$
a_{0} a_{j} . t s=a_{2} a_{j+2}= \begin{cases}a_{0} a_{j+2}-a_{0} a_{2}, & j \neq m-2, \\ -a_{0} a_{2}, & j=m-2 .\end{cases}
$$

Now define vectors

$$
b_{0}=-\frac{1}{m} \sum_{j=1}^{m-1} a_{0} a_{j}
$$

and, for $j=1, \ldots, m-1$,

$$
b_{j}=a_{0} a_{j}+b_{0} .
$$

Then $b_{0} . s=b_{0}$ and $b_{j} . s=b_{m-j}$ for $j=1, \ldots, m-1$. Moreover, $b_{j} . t s=b_{j+2}$ for $j=0, \ldots, m-1$, with indices reduced $\bmod m$ if necessary. Hence the map $a_{j} \mapsto b_{j}$ 
is a $W$-equivariant bijection from the basis $\left\{a_{j}: j=0, \ldots, m-1\right\}$ of the degree 1 component to a spanning set $\left\{b_{j}: j=0, \ldots, m-1\right\}$ of $A_{[S]}$. Clearly, $\sum_{j=0}^{m-1} b_{j}=0$ and so the character of $W$ on $A_{[S]}$ is $\pi_{\mathcal{A}}-1_{S}$.

Lemma 5.9 The element $a_{0} a_{m-1}$ generates the top component $A_{[S]}$ as $\mathbb{C} W$-module.

Proof Let $M=a_{0} a_{m-1} . \mathbb{C} W$. Then $M$ contains the elements

$$
a_{0} a_{1}=a_{0} a_{m-1} . s, \quad a_{1} a_{2}=-a_{0} a_{m-1} . t s, \quad a_{0} a_{2}=a_{0} a_{1}+a_{1} a_{2},
$$

and, by induction, the elements

$$
a_{j-1} a_{j}=a_{j-3} a_{j-2 . t s}, \quad \text { and } \quad a_{0} a_{j}=a_{0} a_{j-1}+a_{j-1} a_{j},
$$

for $j>2$. Consequently, $M$ contains the basis $\left\{a_{0} a_{j}: j=1, \ldots, m-1\right\}$ of $A_{[S]}$, whence $M=A_{[S]}$.

Proposition 5.10 $\Psi_{S}=\Phi_{S} \epsilon_{S}$

Proof We distinguish two cases.

If $m$ is odd, then $\pi_{\mathcal{A}}=\operatorname{Ind}_{\langle s\rangle}^{W}(1)$, since $C_{W}(s)=\langle s\rangle$ and all reflections are conjugates of $s$. Hence

$$
\Psi_{S}=\operatorname{Ind}_{\langle s\rangle}^{W}(1)-1_{S}=\operatorname{Ind}_{\left\langle w_{0}\right\rangle}^{W}(1)-1_{S}=\Phi_{S}
$$

and $\Phi_{S}=\Phi_{S} \epsilon_{S}$, since $\Phi_{S}(w)=0$ for all $w \in W$ with $\epsilon_{S}(w)=-1$.

If $m$ is even, then $\operatorname{Ind}_{\left\langle w_{0}\right\rangle}^{W}(1) \epsilon_{S}=\operatorname{Ind}_{\left\langle w_{0}\right\rangle}^{W}(1)$ and

$$
\Phi_{S} \epsilon_{S}=\left(\operatorname{Ind}_{\left\langle w_{0}\right\rangle}^{W}(1)-1_{S}\right) \epsilon_{S}=\operatorname{Ind}_{\left\langle w_{0}\right\rangle}^{W}(1)-\epsilon_{S}=\pi_{\mathcal{A}}-1_{S}=\Psi_{S},
$$

since $\pi_{\mathcal{A}}-\operatorname{Ind}_{\left\langle w_{0}\right\rangle}^{W}(1)=1_{S}-\epsilon_{S}$, as can be easily verified.

We can now conclude that Conjecture B holds for $W$ of rank 2.

Theorem 5.11 Let $W$ be a Coxeter group of rank 2, generated by $S=\{s, t\}$. Then, with notation as above, the top component characters of $W$ are $\Phi_{S}=\operatorname{Ind}_{\left\langle w_{0}\right\rangle}^{W}(1)-1_{S}$ and $\Psi_{S}=\pi_{\mathcal{A}}-1_{S}=\Phi_{S} \epsilon_{S}$. Moreover, $W$ satisfies Conjecture $B$ with $\varphi_{(s t)}{ }^{j}=\chi_{j}$ in case $m$ odd, while $\varphi_{w_{0}}=\epsilon_{S}$ and $\varphi_{(s t)^{j}}=\chi_{2 j}$ in case $m$ even.

Proof Apply Propositions 5.6, 5.7, and 5.10, and Remark 4.3.

Corollary 5.12 Suppose that $W$ is a Coxeter group with rank at most 2 . Then Conjecture A holds for W.

Proof By Lemmas 5.1 and 5.2, and Theorem 5.11, Conjecture B holds for all parabolic subgroups of $W$. By Theorem 4.4, it suffices to show that Conjecture C holds for all subsets $L \subseteq S$. If $|L|=0,1$, this follows from Corollary 5.3. It follows 
from Theorem 5.11 that Conjecture $\mathrm{C}$ holds when the rank of $W$ and $|L|$ are both equal 2.

It follows in particular from Corollary 5.12 that every Coxeter group of type $I_{2}(\mathrm{~m})$ satisfies Conjecture A. We list the corresponding decomposition of the regular character $\rho_{W}$ into characters $\Phi_{[L]}=\operatorname{Ind}_{N_{W}\left(W_{L}\right)}^{W} \widetilde{\Phi}_{L}$ and the decomposition of the OrlikSolomon character $\omega_{W}$ into characters $\Psi_{[L]}=\operatorname{Ind}_{N_{W}\left(W_{L}\right)}^{W} \widetilde{\Psi}_{L}$ in Table 1 below. In Table 1, the left character table covers the case $m=2 k$ and the right character table covers the case $m=2 k+1$. The columns of the character tables are labeled by representatives of the conjugacy classes of $W$, where the parameter in $(s t)^{i}$ is $i=1, \ldots, k-1$ for $m=2 k$, and $i=1, \ldots, k$ for $m=2 k+1$. An entry ' .' in the table stands for the value 0. As observed in Proposition 5.8, the rank 1 component of $\omega_{W}$ is the permutation character of the action of $W$ on the set $\mathcal{A}$ of hyperplanes. In case $m=2 k$, the constituent $\Psi_{[\{s\}]}$ corresponds to the action on the $W$-orbit of the hyperplane $H_{s}$, and whether the element $s$ has 2 or 1 fixed points in this action depends on whether $k$ is even or odd. In such a situation, an entry of the form ' $x \mid y$ ' in the table stands for ' $x$ if $k$ is even and $y$ if $k$ is odd'.

We saw in Theorem 5.11 that Conjecture B holds when $W$ has rank 2 and we saw in Corollary 5.3 that Conjecture C holds when the subset $L \subseteq S$ has size $|L| \leq 1$. In the rest of this section, we prove that if the parabolic subgroup $W_{L}$ has rank 2, then Conjecture $\mathrm{C}$ holds for any overgroup $W$. A similar result when $W_{L}$ is a product of symmetric groups would reduce the proof of Conjecture A to considering only a small number of cases.

From now on, $W$ is a finite Coxeter group, generated by $S$ with $|S| \geq 3$ and $W_{L}$ is a rank 2 parabolic subgroup of $W$ with $L=\{s, t\} \subseteq S$. The elements $x_{K}$ and $e_{K}$ are defined relative to the ambient set $S$. We use a superscript to indicate this ambient set when it is not equal to $S$. Thus, for $K \subseteq L, x_{K}^{L}$ denotes a basis element of the descent algebra of $W_{L}$.

If $W_{L}$ is bulky, then $W_{L}$ satisfies Conjecture C, by Theorem 4.7.

Suppose $W_{L}$ is not bulky. Then $N_{L}$ does not centralize $W_{L}$ and so $N_{L}$ contains an element inducing the nontrivial graph automorphism $\gamma$ on $W_{L}$, interchanging $s$ and

Table 1 The characters $\Phi_{\lambda}$ and $\Psi_{\lambda}$ for $I_{2}(m) ; m=2 k, m=2 k+1$

\begin{tabular}{|c|c|c|c|c|c|c|c|c|c|}
\hline & 1 & $s$ & $t$ & $w_{0}$ & $(\mathrm{st})^{i}$ & & 1 & $s$ & $(\mathrm{st})^{i}$ \\
\hline$\Phi_{[\varnothing]}$ & 1 & 1 & 1 & 1 & 1 & $\Phi_{[\varnothing]}$ & 1 & 1 & 1 \\
\hline$\Phi_{[\{s\}]}$ & $k$ &. $\mid 1$ & $\cdot \mid-1$ & $-k$ & . & $\Phi_{[\{s\}]}$ & $m$ & -1 & . \\
\hline$\Phi_{[\{t\}]}$ & $k$ & $\cdot \mid-1$ &. $\mid 1$ & & . & $\Phi_{[S]}$ & $m-1$ & . & -1 \\
\hline$\Phi_{[S]}$ & $m-1$ & -1 & -1 & $m-1$ & -1 & & & & \\
\hline$\rho_{W}$ & $2 m$ & . & . & . & . & $\rho_{W}$ & $2 m$ & . &. \\
\hline$\Psi_{[\varnothing]}$ & 1 & 1 & 1 & 1 & 1 & $\Psi_{[\varnothing]}$ & 1 & 1 & 1 \\
\hline$\Psi_{[\{s\}]}$ & $k$ & $2 \mid 1$ &. $\mid 1$ & $k$ & . & $\Psi_{[\{s\}]}$ & $m$ & 1 & . \\
\hline$\Psi_{[\{t\}]}$ & $k$ &. $\mid 1$ & $2 \mid 1$ & $k$ & . & $\Psi_{[S]}$ & $m-1$ & . & -1 \\
\hline$\Psi_{[S]}$ & $m-1$ & 1 & 1 & $m-1$ & -1 & & & & \\
\hline$\omega_{W}$ & $2 m$ & 4 & 4 & $2 m$ & . & $\omega_{W}$ & $2 m$ & 2 & . \\
\hline
\end{tabular}


$t$. In this case, $s$ and $t$ are conjugate in $W$ and so $W_{L}$ is either of type $A_{1} \times A_{1}$ or of type $I_{2}(m)$ for odd $m>2$. We distinguish two cases accordingly.

First, suppose that $W_{L}$ is of type $A_{1} \times A_{1}$. Then $W_{L}$ has exactly one cuspidal element $w=s t=t s$, which is central in $W_{L}$ and invariant under $N_{L}$, hence central in $N_{W}\left(W_{L}\right)$. We have

$$
\varphi_{w}=\Phi_{L}=\epsilon_{L}, \quad \text { and } \quad \psi_{w}=\Psi_{L}=1_{L},
$$

by Corollary 5.5 and Proposition 5.8. Parts (i) and (ii) of Conjecture C are therefore trivially satisfied, with

$$
\widetilde{\varphi}_{w}=\widetilde{\Phi}_{L}, \quad \text { and } \quad \widetilde{\psi}_{w}=\widetilde{\Psi}_{L}
$$

which exist by Propositions 2.4 and 3.1.

For part (iii) of Conjecture $\mathrm{C}$, note that the idempotent

$$
f=\frac{1}{4}(1-s-t+s t)
$$

spans a subspace of $\mathbb{C} W_{L}$ affording the character $\Phi_{L}$. As in the proof of Lemma 5.4,

$$
e_{L}^{L}=1-\frac{1}{2} x_{s}^{L}-\frac{1}{2} x_{t}^{L}+\frac{1}{4} x_{\varnothing}^{L}=\frac{1}{4}(1+s t)-\frac{1}{4}(s+t)=f,
$$

and thus $e_{L}^{L} f=e_{L}^{L}$ is a basis of the top component of $W_{L}$ which is centralized by $N_{L}$. Hence $\widetilde{\varphi}_{w}(u n)=\varphi_{w}(u)$, for $u \in W_{L}$ and $n \in N_{L}$. Moreover, note that $a_{L}=a_{s} a_{t}$ spans the top component of $A\left(W_{L}\right)$, and that $e_{L} n=e_{L}$, whereas $a_{L} \cdot n=\sigma_{L}(n) a_{L}$ for $n \in N_{L}$. It follows that $\widetilde{\psi}_{L}(u n)=\psi_{L}(u) \sigma_{L}(n)=\varphi_{L}(u) \epsilon(u) \epsilon(n) \alpha_{L}(n)=$ $\widetilde{\varphi}_{L}(u n) \epsilon(u n) \alpha_{L}(u n)$, for $u \in W_{L}$ and $n \in N_{L}$, as desired. This proves the following proposition.

Proposition 5.13 Suppose $L=\{s, t\} \subseteq S$ is such that $W_{L}$ is of type $A_{1} \times A_{1}$. Then Conjecture C holds for $L \subseteq S$.

Second, suppose that $W_{L}$ is of type $I_{2}(m)$ where $m=2 k+1$. Recall the characters $\chi_{j}: s t \mapsto \zeta_{m}^{j}$ for $j=1, \ldots, m-1$. The centrally primitive idempotent in $\mathbb{C}\langle s t\rangle$ affording $\chi_{j}$ is

$$
f_{j}=\frac{1}{m} \sum_{k=0}^{m-1} \zeta_{m}^{j k}(s t)^{-k}
$$

The cuspidal conjugacy classes in $W_{L}$ are represented by $c_{j}=(s t)^{j}$ for $j=1, \ldots, k$ and $C_{W_{L}}\left(c_{j}\right)$ is the rotation subgroup $W^{+}=\langle s t\rangle$ of $W$. Moreover, for $j=1, \ldots, k$ the characters $\varphi_{(s t)^{j}}=\chi_{j}$ satisfy the conclusions of Conjecture B for $W_{L}$ and the line $\mathbb{C} e_{L}^{L} f_{j}$ in $e_{L}^{L} \mathbb{C} W_{L}$ affords the character $\varphi_{(s t)^{j}}$ of $W^{+}$. As usual, denote by $w_{L}$ the longest element of $W_{L}$. Note that $f_{j}^{w_{L}}=f_{m-j}$, for $j=1, \ldots, k$, since $(s t)^{w_{L}}=(s t)^{-1}$, and that $e_{L}^{L} f_{j}=\operatorname{Av}\left(\left\langle w_{L}\right\rangle\right) f_{j}$, by Lemma 5.4, since $\operatorname{Av}\left(W_{L}\right) f_{j}=$ $\sum_{k=0}^{m-1} \zeta_{m}^{j k} \operatorname{Av}\left(W_{L}\right)=0$, for $j=1, \ldots, m-1$. Obviously, the graph automorphism $\gamma$ 
swaps $e_{L}^{L} f_{j}$ and $e_{L}^{L} f_{m-j}$, as does right multiplication by $w_{L}$ :

$$
\begin{aligned}
e_{L}^{L} f_{j} w_{L} & =\operatorname{Av}\left(\left\langle w_{L}\right\rangle\right) f_{j} w_{L}=\operatorname{Av}\left(\left\langle w_{L}\right\rangle\right) w_{L} f_{j}^{w_{L}} \\
& =\operatorname{Av}\left(\left\langle w_{L}\right\rangle\right) f_{j}^{w_{L}}=\operatorname{Av}\left(\left\langle w_{L}\right\rangle\right) f_{m-j}=e_{L}^{L} f_{m-j} .
\end{aligned}
$$

Recall from [4, Lemma 3.2] that $N_{L}$ centralizes $e_{L}^{L}$ and that $N_{W}\left(W_{L}\right)$ acts naturally on $\mathbb{C} W_{L}$ with $a . n w=n^{-1} a n w$ for $a$ in $\mathbb{C} W_{L}, n$ in $N_{L}$, and $w$ in $W_{L}$. Suppose $z$ is in $C_{W}\left(c_{j}\right)$. Then $z=n w$, where $n$ is in $N_{L}$ and $w$ is in $W_{L}$. If $n$ centralizes $W_{L}$, then

$$
e_{L}^{L} f_{j} \cdot z=n^{-1} e_{L}^{L} f_{j} n w=e_{L}^{L} f_{j} w
$$

and $w$ is in $W^{+}$. If $n$ does not centralize $W_{L}$, then $n w_{L}$ centralizes $W_{L}$ and $n w=$ $\left(n w_{L}\right)\left(w_{L} w\right)$, so

$$
e_{L}^{L} f_{j} \cdot z=\left(w_{L} n^{-1}\right) e_{L}^{L} f_{j}\left(n w_{l}\right)\left(w_{L} w\right)=e_{L}^{L} f_{j}\left(w_{L} w\right)
$$

and $w_{L} w$ is in $W^{+}$. It follows that the characters $\varphi_{(s t)^{j}}$ of $W^{+}$extend to characters $\widetilde{\varphi}_{(s t)^{j}}$ of the centralizers $C_{W}\left(c_{j}\right)$ with

$$
\tilde{\varphi}_{(s t)^{j}}(z)=\varphi_{(s t)^{j}}(v),
$$

where if $z=n w$ with $n \in C_{W}\left(W_{L}\right)$, then $v=w$, and if $z=n w$ with $n \notin$ $C_{W}\left(W_{L}\right)$, then $v=w_{L} w$. For $j=1, \ldots, k$, define $M_{j}=e_{L}^{L} f_{j} \mathbb{C} W_{L}$. Then $M_{j}$ is a $N_{W}\left(W_{L}\right)$-module with basis $\left\{e_{L}^{L} f_{j}, e_{L}^{L} f_{m-j}\right\}$ and character $\operatorname{Ind}_{C_{W}\left(c_{j}\right)}^{N_{W}\left(W_{L}\right)} \tilde{\varphi}_{(s t)}$. Clearly, $e_{L}^{L} \mathbb{C} W_{L} \cong \bigoplus_{j=1}^{k} M_{j}$ is a decomposition of $e_{L}^{L} \mathbb{C} W_{L}$ as a direct sum of $N_{W}\left(W_{L}\right)$ modules. By [4, Corollary 3.13], the character of $e_{L}^{L} \mathbb{C} W_{L}$ is $\widetilde{\Phi}_{L}$, and so we conclude that

$$
\widetilde{\Phi}_{L}=\sum_{j=1}^{k} \operatorname{Ind}_{C_{W}\left(c_{j}\right)}^{N_{W}\left(W_{L}\right)} \widetilde{\varphi}_{(s t)^{j}} .
$$

Thus, part (i) of Conjecture $\mathrm{C}$ holds. By Remark 4.3, to show that Conjecture $\mathrm{C}$ holds, it suffices to show that $\widetilde{\Psi}_{L}=\widetilde{\Phi}_{L} \epsilon_{S} \alpha_{L}$. Define $a_{L}=a_{s} a_{t}$ in $A(W)$, and recall from Lemma 5.9 that $a_{L} \mathbb{C} W_{L}$ is isomorphic to the top component of $A\left(W_{L}\right)$. Since $m$ is odd, we have $W_{L}=W^{+} \cup w_{L} W^{+}$and thus

$$
a_{L} \mathbb{C} W^{+}=a_{L} \mathbb{C} W_{L},
$$

since $a_{L} w_{L}=a_{s} a_{t} \cdot w_{L}=a_{t} a_{s}=-a_{s} a_{t}=-a_{L}$. Define $f_{0}=\operatorname{Av}\left(W^{+}\right)$. Then the idempotents $f_{j}$ for $j=0, \ldots, m-1$ form a Wedderburn basis of the group algebra $\mathbb{C} W^{+}$and so the module $a_{L} \mathbb{C} W^{+}$is spanned by the elements $\left\{a_{L} f_{j}: j=\right.$ $0, \ldots, m-1\}$. Since

$$
\begin{aligned}
a_{L} f_{0} & =\sum_{k=0}^{m-1} a_{0} a_{m-1} \cdot(t s)^{k}=\sum_{k=0}^{m-1} a_{k+1} a_{k} \\
& =a_{0} a_{m-1}-a_{0} a_{1}+\sum_{k=1}^{m-2} a_{0} a_{k}-a_{0} a_{k+1}=0
\end{aligned}
$$


we see that $\left\{a_{L} f_{j}: j=1, \ldots, m-1\right\}$ is a $\mathbb{C}$-basis of $a_{L} \mathbb{C} W_{L}$. By construction $f_{j} s t=$ $\zeta_{m}^{j} f_{j}$ and by definition $\epsilon(s t)=\alpha_{L}(s t)=1$. Therefore,

$$
a_{L} f_{j} . s t=\zeta_{m}^{j} a_{L} f_{j} \quad \text { and } \quad e_{L}^{L} f_{j} . s t=\epsilon(w) \alpha_{L}(w) \zeta_{m}^{j} e_{L}^{L} f_{j} .
$$

We have seen that $f_{j} w_{L}=w_{L} f_{m-j}, a_{L} \cdot w_{L}=-a_{L}$, and in Lemma 5.4 that $e_{L}^{L} w_{L}=$ $e_{L}^{L}$. Also, $\epsilon\left(w_{L}\right)=-1$, and $\alpha_{L}\left(w_{L}\right)=1$. Therefore,

$$
a_{L} f_{j} \cdot w_{L}=a_{L} f_{m j} \quad \text { and } \quad e_{L}^{L} f_{j} \cdot w_{L}=\epsilon\left(w_{L}\right) \alpha_{L}\left(w_{L}\right) e_{L}^{L} f_{m-j} .
$$

For $n$ in $N_{L}$, we have $f_{j} . n=n f_{j}^{n}, a_{L} n=\sigma_{L}(n) a_{L}$, where by Lemma $2.1 \sigma_{L}(n)=$ $\epsilon(n) \alpha_{L}(n)$, and $e_{L}^{L} \cdot n=n^{-1} e_{L}^{L} n=e_{L}^{L}$. Therefore,

$$
a_{L} f_{j} . n=\sigma_{L}(n) a_{L} f_{j}^{n} \quad \text { and } \quad e_{L}^{L} f_{j} . n=\epsilon(n) \alpha_{L}(n) \sigma_{L}(n) e_{L}^{L} f_{j}^{n} .
$$

Because $N_{W}\left(W_{L}\right)$ is generated by $s t, w_{L}$, and $N_{L}$, it follows from (5.14), (5.15), and (5.16) that $\widetilde{\Psi}_{L}(w)=\widetilde{\Phi}_{L}(w) \epsilon_{S}(w) \alpha_{L}(w)$ for $w$ in $N_{W}\left(W_{L}\right)$. This proves the following proposition.

Proposition 5.17 Suppose $L=\{s, t\} \subseteq S$ is such that the order $m$ of st is odd. Then Conjecture $C$ holds for $L \subseteq S$.

We summarize Propositions 5.13, 5.17, and Theorem 4.7 for rank 2 parabolic subgroups as follows.

Theorem 5.18 Suppose that $W_{L}$ is a rank 2 parabolic subgroup of $W$. Then Conjecture C holds for $W_{L}$.

Acknowledgements The authors acknowledge the financial support of the DFG-priority programme SPP1489 "Algorithmic and Experimental Methods in Algebra, Geometry, and Number Theory". Part of the research for this paper was carried out while the authors were staying at the Mathematical Research Institute Oberwolfach supported by the "Research in Pairs" programme in 2010. The second author wishes to acknowledge support from Science Foundation Ireland.

\section{References}

1. Bergeron, F., Bergeron, N., Garsia, A.M.: Idempotents for the free Lie algebra and $q$-enumeration. In: Invariant Theory and Tableaux, Minneapolis, MN, 1988, IMA Vol. Math. Appl., vol. 19, pp. 166-190. Springer, New York (1990)

2. Bergeron, F., Bergeron, N., Howlett, R.B., Taylor, D.E.: A decomposition of the descent algebra of a finite Coxeter group. J. Algebr. Comb. 1(1), 23-44 (1992)

3. Brieskorn, E.: Sur les groupes de tresses [d'après V. I. Arnol'd]. In: Séminaire Bourbaki, 24ème année (1971/1972), Exp. No. 401, Lecture Notes in Math., vol. 317, pp. 21-44. Springer, Berlin (1973)

4. Douglass, J.M., Pfeiffer, G., Röhrle, G.: Coxeter arrangements and Solomon's descent algebra. arXiv:1101.2075 (2011)

5. Geck, M., Pfeiffer, G.: Characters of Finite Coxeter Groups and Iwahori-Hecke Algebras. London Mathematical Society Monographs. New Series, vol. 21. Clarendon Press, New York (2000)

6. Hanlon, P.: The action of $S_{n}$ on the components of the Hodge decomposition of Hochschild homology. Mich. Math. J. 37(1), 105-124 (1990) 
7. Howlett, R.B.: Normalizers of parabolic subgroups of reflection groups. J. Lond. Math. Soc. 21, 62-80 (1980)

8. Konvalinka, M., Pfeiffer, G., Röver, C.: A note on element centralizers in finite Coxeter groups. J. Group Theory (2011). doi:10.1515/JGT.2011.074, arXiv:1005.1186

9. Lehrer, G.I., Solomon, L.: On the action of the symmetric group on the cohomology of the complement of its reflecting hyperplanes. J. Algebra 104(2), 410-424 (1986)

10. Orlik, P., Solomon, L.: Coxeter arrangements. In: Singularities, Part 2, Arcata, Calif., 1981. Proc. Sympos. Pure Math., vol. 40, pp. 269-291. Amer. Math. Soc, Providence (1983)

11. Orlik, P., Terao, H.: Arrangements of Hyperplanes. Grundlehren der Mathematischen Wissenschaften, vol. 300. Springer, Berlin, (1992)

12. Pfeiffer, G.: A quiver presentation for Solomon's descent algebra. Adv. Math. 220(5), 1428-1465 (2009)

13. Pfeiffer, G., Röhrle, G.: Special involutions and bulky parabolic subgroups in finite Coxeter groups. J. Aust. Math. Soc. 79(1), 141-147 (2005)

14. Schocker, M.: Über die höheren Lie-Darstellungen der symmetrischen Gruppen. Bayreuth. Math. Schr. 63, 103-263 (2001)

15. Solomon, L.: A Mackey formula in the group ring of a Coxeter group. J. Algebra 41(2), 255-264 (1976) 